\title{
Mladen J. Žujović et le mouvement de résistance de Dragoljub Mihailović
}

Mladen J. Žujović and the resistance movement of Dragoljub Mihailović

\section{Roland Vasic}

\section{(2) OpenEdition}

\section{Journals}

Édition électronique

URL : https://journals.openedition.org/ceb/754

DOI : $10.4000 /$ ceb.754

ISSN : 2261-4184

Éditeur

INALCO

\section{Édition imprimée}

Date de publication : 30 mars 2011

Pagination : 19-36

ISBN : 978-2-85831-189-7

ISSN : 0290-7402

Référence électronique

Roland Vasic, « Mladen J. Žujović et le mouvement de résistance de Dragoljub Mihailović », Cahiers balkaniques [En ligne], 38-39 | 2011, mis en ligne le 17 février 2012, consulté le 06 juillet 2021. URL : http://journals.openedition.org/ceb/754 ; DOl : https://doi.org/10.4000/ceb.754

Ce document a été généré automatiquement le 6 juillet 2021.

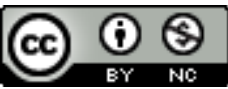

Cahiers balkaniques est mis à disposition selon les termes de la Licence Creative Commons Attribution - Pas d'Utilisation Commerciale 4.0 International. 


\title{
Mladen J. Žujović et le mouvement de résistance de Dragoljub Mihailović
}

\author{
Mladen J. Žujović and the resistance movement of Dragoljub Mihailović
}

\author{
Roland Vasic
}

1 La Seconde Guerre mondiale menaçant à ses portes, le royaume de Yougoslavie a dû réaménager son organisation intérieure pour satisfaire des aspirations nationales centrifuges et tenter de sauvegarder son intégrité.

2 L'accord du 26 août 1939, dit "Sporazum », entre le chef du gouvernement Dragiša Cvetković et le leader du peuple croate, chef du parti paysan, Vlatko Maček, a été promu par le prince régent Paul Karageorgévitch, soucieux, dès son accession au pouvoir en 1934, de trouver une solution à la « question croate ». Il a permis la création d'une entité, la « Banovine de Croatie ». La transformation de la Yougoslavie centralisée en un État fédéral n'est cependant pas l'objet d'un consensus suffisant de la part des représentants principaux des peuples serbe, croate et slovène. Leurs rivalités, focalisées sur la domination de territoires à populations mixtes, semblent ravivées par la mise en œuvre d'une politique destinée justement à sauver l'État commun. L'opinion serbe, en grande partie, conteste la délimitation de la «Banovine de Croatie», et estime nécessaire que soient réaffirmés ses intérêts particuliers. L'idéologie $d u$ "yougoslavisme» unitaire ne guide quasiment plus personne, mais la mutation fédérale, voire confédérale, est entreprise de façon déséquilibrée, dans un climat de surenchère et de suspicion.

3 Par ailleurs, le régent Paul est soumis à la pression des conquêtes et des influences allemandes et italiennes présentes dans tout l'environnement proche, à l'exception de la Grèce. Anglophile, il sait ne pouvoir compter sur un soutien suffisant de Londres et cède à Hitler, en signant l'adhésion au Pacte tripartite, le 25 mars 1941, à Vienne. L'accord semble avoir prévu de ne pas engager la Yougoslavie sur le champ de bataille. Hitler s'est montré intéressé par le maintien d'un pays uni et quasi-neutre, source future d'approvisionnement et zone de passage libre. En vérité, il ne conçoit pas de 
refus. La tension des relations serbo-croates est également une faiblesse majeure de l'État yougoslave dans sa politique étrangère.

4 L'opinion serbe, ses principaux partis et les figures de l'Église orthodoxe réagissent contre ce pacte. Il en est de même dans les secteurs pro-britanniques de l'institution militaire. Dans la nuit du 26 au 27 mars, un putsch est mené par le général Borivoj Mirković. Le régent Paul est contraint de se retirer et le jeune roi Pierre est proclamé majeur. À Belgrade et en province (dans les principales villes de Serbie, du Monténégro ainsi que Sarajevo, Skopje, Split et Ljubljana), la rue (où se retrouvent étudiants, sympathisants pro-occidentaux, en majorité pro-yougoslaves et serbes, ainsi que membres du parti communiste et de ses sections de jeunesse) manifeste son soutien par les slogans :

«Mieux vaut la guerre que le pacte! Mieux vaut la tombe que l'esclavage! Dehors Hitler!

Vive la liberté !" "

5 Le général Dušan Simović forme un gouvernement de large rassemblement avec Maček (que Londres encourage à accepter et qui pose, entre autres, comme condition, la non dénonciation du Pacte). Une volonté domine de tempérer les relations avec Berlin et Rome, comme si le camouflet pouvait être occulté. L'état-major général veut mobiliser, mais le nouveau gouvernement entend parlementer, gagner du temps. Le ministère de la guerre tergiverse. Hitler, lui, a ordonné, dès le 27, d'attaquer la Yougoslavie. Il entend la "punir ", sans prendre la peine de lui déclarer la guerre. Le bombardement de Belgrade, le matin du 6 avril, inaugure un rapide assaut du pays. Il marque aussi, de la part du Führer, une volonté de traiter la partie serbe de la façon la plus brutale. L'ordre de mobilisation générale de l'armée yougoslave est enfin lancé le 7. La " guerre d'avril » a été conçue rapidement par Berlin. Elle doit être menée de la même manière. L'opposition fournie par l'armée yougoslave est inégale, beaucoup trop faible pour soutenir quelque front solide. La défaite est consommée en dix jours à peine.

6 Le 15 avril, le haut commandement est arrêté sans difficulté par les Allemands, dans les environs de Sarajevo. Deux jours plus tard, le général Kalafatović signe à Belgrade une capitulation sans condition. Le roi et quasiment tous les membres du gouvernement sont, alors, déjà partis pour la Grèce. Maček, qui a quitté le gouvernement le 8 avril pour Kupinec, près de Zagreb, en compagnie d'autres ministres croates, a appelé, deux jours plus tard, à la loyauté à l'égard du nouveau pouvoir oustachi. Le lien entre l'armée et le pouvoir civil est rompu, le pays rapidement dépecé. Simović, en fait, n'a pas mandaté Kalafatović pour solliciter autre chose qu'une trêve (se référant à la situation de la France en 1940), mais cela sans concertation avec son cabinet ${ }^{2} .375000$ soldats et officiers sont faits prisonniers : Serbes, Monténégrins et Slovènes, ils sont envoyés dans des camps en Allemagne ${ }^{3}$. Les militaires croates se sont peu opposés à l'envahisseur, assez souvent l'ont attendu avec bienveillance. Les germanophones locaux ont de suite rejoint la Wehrmacht. Des hommes échappent à la captivité et vont rejoindre leurs villes ou régions d'origine. Le pays est vite démantelé, au profit de Berlin, Rome, des États voisins, de ceux pour qui, la Yougoslavie, « créature de Versailles », n'était que le cadre d'une " hégémonie grand-serbe ».

7 Le colonel Dragoljub Mihailović (1893-1946), assistant du chef d'état-major de la Deuxième armée au nord de la Bosnie, refuse aussitôt la capitulation.

Le 13 avril, il a eu à organiser une " unité rapide motorisée » afin d'effectuer une mission de reconnaissance. Le 14, il a pris la ville de Derventa, que tenaient des Croates en 
rébellion. À Doboj, le 15, quand le haut commandement est arrêté, il s'est dirigé au sud et a perdu tous ses véhicules dans un accrochage avec une colonne allemande.

Il prend alors le maquis ${ }^{4}$. Le 20 avril, il apprend la capitulation signée trois jours plus tôt, et annonce à ses hommes qu'elle ne l'engage pas. Il entend mener une activité de guérilla, comptant sur le soutien des Britanniques toujours en lutte, conduisant des actions de sabotage de la voie ferrée Zavidovići-Han Pijesak et des équipements de communication. Les Allemands le repèrent. Il est attaqué également par une unité croate et une milice musulmane. Se repliant vers l'est, il passe la rivière Drina séparant la Bosnie de la Serbie, le 29 avril, au nord de Višegrad. En Serbie, il compte trouver un terrain favorable, envisage d'abord de se rendre sur le mont Rudnik, bien situé, à distance relative de Belgrade et des autres villes serbes de Šumadija (région considérée comme un haut lieu des luttes nationales passées). Réunissant à nouveau ses hommes, dont des recrues faites en chemin, il déclare ne vouloir garder avec lui que les volontaires, et libère les autres de leurs obligations le 5 mai (six officiers et une vingtaine d'hommes le quittent). Il se rend alors au village de Struganik pour consulter son ami Aleksandar Mišić, le fils du renommé voïvode ${ }^{5}$ de la Première Guerre mondiale. Son groupe va l'attendre aux alentours, au lieu-dit Ravna Gora (« Montagne plate »), le 11 mai. Il compte alors sept officiers et vingt-quatre hommes dont deux Croates, six Macédoniens et un Juif. Il rejoint ces hommes le 13. C'est ce col, entre les monts Maljen et Suvobor, qui donnera son nom au mouvement. Selon l'un de ses subordonnés :

"Ravna Gora (997) est le bastion ultime au sud-ouest du massif du Suvobor. De ce lieu, il était décidé à donner, encore une fois, une leçon à l'ennemi jaune et noir (couleurs de l'Empire austro-hongrois) et à l'avertir, que pouvait se reproduire le 20 novembre 1914, début de la bataille de Suvobor. ${ }^{6} "$

L'endroit assure une relative sécurité. Il est difficile d'accès aux véhicules motorisés et fournit plusieurs pistes d'évacuation. Mihailović envoie Mišić prendre des contacts à Belgrade, et expose à nouveau ses vues: organiser une armée clandestine avec le soutien britannique puis mener, le moment opportun, à la libération du pays. Les forces de l'Axe se sont imposées rapidement et brutalement. Une lutte frontale n'est déjà plus possible. Le colonel est convaincu de la victoire britannique à terme. Il s'agit de préparer en ce sens la Yougoslavie, de constituer des réseaux avec les militaires disponibles et de gagner la bienveillance de la population. Aucun programme politique n'est formulé.

"Son ambition était d'empêcher une "reddition morale » de son peuple, en maintenant un centre de résistance, qui puisse représenter une Yougoslavie "libre" . " "

Les hommes qui constituent ce mouvement sont rapidement appelés "Tchetniks " (membre d'une "četa ", petite unité, et en référence aux francs-tireurs serbes mobilisés au début du siècle pour lutter contre les autorités turques, puis les komitadjis bulgares et les « irréguliers » albanais en Macédoine et au Kosovo).

Mihailović ne donne donc pas à sa démarche de fondement politique explicite. Il la conçoit comme la poursuite d'un «effort de guerre " interrompu trop vite. «La politique » est bien plutôt associée aux faiblesses qui ont causé l'effondrement de l'État. Les hommes qui vont être ses premiers "conseillers politiques" sont animés d'un esprit assez semblable. Civils, mobilisés en tant qu'officiers de réserve, ils partagent l'idée que la Yougoslavie, sa composante serbe en tout état de cause, ne peut en rester là, ni accepter sans coup férir la domination de l'Axe et de ses alliés.

"Il était clair qu'il ne venait à l'esprit de personne de traiter la question politique. Seuls,

l'idée et le désir, que le peuple, dans son ensemble, s'oppose de toutes ses forces à l'occupant 
et à ses collaborateurs, les portaient. Etant entendu qu'étaient exclus, ceux qui considéraient

les Allemands, vainqueurs, et l'état de fait comme définitif." "

13 Ces dispositions d'esprit sont celles d'un Mladen J. Žujović (1895-1969). Elles se forgent et déterminent son engagement à résister, dans les jours qui suivent l'annonce de la capitulation de l'armée yougoslave et le départ en exil du roi et des principaux dirigeants ${ }^{9}$. Au service de l'état-major du général M. Damjanović, à Prijepolje, quand arrivent les Allemands, le 16 avril, il décide en compagnie de son ami Dragiša Vasić (1885-1945), de ne pas se rendre aux vainqueurs et de quitter la ville. Le lendemain, les deux hommes partent pour la région d'origine de Vasić, en Serbie occidentale ${ }^{10}$. Pendant près de deux mois, Žujović se cache dans la région de Rudnik et s'enquiert de l'évolution de la situation. Informé il se rend au quartier général qu'a établi Dragoljub Mihailović à Ravna Gora, non loin de là. C'est sur son idée, qu'est entreprise alors la formation d'une instance politique propre au mouvement ${ }^{11}$. Il présente au colonel un «Projet de formation et d'organisation d'un organe exécutif civil et politique sur lequel aura à s'appuyer la branche militaire du mouvement $»^{12}$. La proposition de constituer un collectif de personnalités de la vie politique, de la vie culturelle et des sociétés patriotiques, ainsi qu'un représentant de l'état-major de Mihailović, est acceptée début août. Jusquelà, et ce depuis la mi-mai, un état-major clandestin œuvre dans la capitale, réunit des fonds et espionne notamment les télécommunications.

Žujović est désigné comme membre du nouvel organe, baptisé par le colonel «Comité central national » (CNK en serbe). Il est rapidement envoyé à Belgrade pour l'y fonder et organiser. Chemin faisant, il va rencontrer Vasić, aux environs de Gornji Milanovac, afin de " partager avec lui ses impressions et espoirs ${ }^{13}$ ". Vasić rejoint à la mi-août Dragoljub Mihailović à Ravna Gora. Il s'y installe pour devenir ensuite le conseiller politique principal du mouvement.

15 À Belgrade, les principaux partis politiques ont vu leurs leaders prendre le chemin de l'exil, en tant que membres du gouvernement issu du pronunciamiento qui a défié Hitler. Ce sont Momčilo Ninčić et Miloš Trifunović du Parti radical, Milan Grol et Boža Marković du Parti démocrate, Srđan Budisavljević du Parti démocrate indépendant, Bogoljub Jevtić et Petar Živković du Parti national yougoslave ainsi que Milan Gavrilović de l'Union agrarienne, appelé comme ambassadeur à Moscou. Juraj Krnjević et Josip Šutej représentent, dans le gouvernement en exil, le Parti paysan croate.

16 La population est sous le choc d'un abandon de fait aux soldats et cadres allemands. L'opinion désemparée, quand bien même certains entretiennent une forme d'espoir en écoutant Radio Londres, voit de suite toute expression interdite.

"Une grande part du courant bourgeois antifasciste se replia, se protégea au travers un comportement discret, une indifférence pour la politique, en vivant avec peine les souffrances serbes..$^{14}$ "

17 Le gouvernement des «commissaires » de l'ex-ministre de l'intérieur Milan Aćimović, formé le $1^{\mathrm{er}}$ mai, assume la position de pendant civil de l'autorité militaire allemande. Il est composé de membres de formations de faible audience, de quelques personnalités liées, comme Aćimović, à l'ancien premier ministre Stojadinović (rare Serbe que les autorités allemandes semblent estimer), ou à Dimitrije Ljotić («disciple» local de Charles Maurras et le plus idéologiquement déterminé en faveur du «nouvel ordre européen »). D'autres germanophiles, plus en vue, le complèteront en juillet autour du général Nedić1 ${ }^{15}$. 
Žujović a donc fort à faire pour constituer et animer le nouveau CNK. Né à Belgrade, le 5 juin 1895, d'un père médecin-chef d'une unité hospitalière de la capitale, élève de l'Académie militaire ( $46^{\mathrm{ème}}$ classe), il a été mobilisé lors de la Première Guerre mondiale, évacué à Corfou en janvier 1916, puis s'est battu sur le Front de Salonique, a été décoré. En 1920, il a quitté l'armée pour protester contre la promotion jugée illégitime de militaires qui avaient servi dans l'armée austro-hongroise. Il a entrepris des études de droit à Paris et obtenu une thèse, en 1928, avec pour sujet : « Le pouvoir constituant dans les constitutions serbes ${ }^{16}$. À défaut de la carrière diplomatique qu'il souhaitait, il a ouvert en 1932, un cabinet d'avocats à Belgrade, avec son ami (depuis mars 1915) Dragiša Vasić. Membre, comme lui, du « comité principal » du Parti républicain, il s'est engagé plus vivement sur le devant de la scène quand il a adhéré au Club culturel serbe en 1937. Francophile, il a été décoré de la Croix de chevalier de la Légion d'honneur en 1936, pour son activité au sein de la «Société des amis de la France ». Il est mobilisé en avril 1941, comme lieutenant de réserve d'infanterie.

19 À l'instar de Mladen J. Žujović, chargé d'organiser l'instance politique officielle du mouvement, il faut noter l'appartenance des principales personnalités autour de Mihailović (dont l'orientation personnelle se rapproche plutôt des agrariens) à une formation de modeste assise électorale : le Parti républicain, dont sont membres Dragiša Vasić, Vojislav Vujanac et Jovan Đonović, futurs cadres du mouvement de Mihailović.

Ce parti, composé principalement d'intellectuels et d'universitaires hostiles au principe monarchique perçu comme "...défenseur naturel et historique de la richesse illimitée et des minorités privilégiées ${ }^{17}$ et convaincus de la nécessité de dissocier la dynastie de l'avenir de l'Etat yougoslave, n'a pas de base électorale étendue. L'enseignant, écrivain et député, Jaša Prodanović (qui rejoindra Tito en 1944) et le philologue, ancien chef de gouvernement et ministre de l'éducation, Ljubomir Stojanović en sont les deux figures principales, à sa création en tant que Parti républicain démocrate, en janvier 1920. L'année suivante, il devient Parti républicain yougoslave et se détermine pour une organisation fédérale du pays, reconnaissant les individualités serbe, croate et slovène et exprimant le souci d'un équilibrage de la domination démographique des Serbes (Stojanović reconnaît également la spécificité de la langue macédonienne et propose un statut d'autonomie pour la région, considérée à Belgrade comme "Serbie du sud»), le suffrage universel direct à bulletin secret, l'indépendance de la justice, l'égalité hommes-femmes, la limitation de la journée de travail, la protection de femmes et enfants au travail. On lit dans son programme :

"Le régime républicain et un ordre social juste dans notre nouvel Etat yougoslave! En d'autres mots, la liberté politique et la justice sociale. Le parti républicain démocrate est contre la révolution sociale mais pour une réforme agraire profonde, pour la protection des travailleurs face à l'exploitation excessive du capital, la destruction du spahiluk ${ }^{18}$; défenseur des pleines libertés démocratiques, de la souveraineté totale du peuple dans l'Etat : le PRD est aussi pacifiste, adversaire des conflits armés entre les peuples... ${ }^{19}$ "

21 Ce parti n'a pas subi les foudres particulières du régime autoritaire entre 1929 et 1935, $\mathrm{du}$ fait notamment de son expression principale dans les salons et les cercles académiques. On note la proximité de nombre de ses membres avec les milieux s'inscrivant dans la filiation de l'organisation secrète nationaliste "L'union ou la mort " dite aussi "la Main noire " (liée à l'attentat de Sarajevo en 1914 et dont le leader Dimitrijević dit Apis a été condamné à mort par les autorités serbes en 1917). La revue Republika est toutefois interdite depuis 1925. Žujović, Vasić, Vujanac et Đonović appartiennent à la jeune génération de ces républicains. 
Leaders républicains, agrariens et autres se retrouvent à l'avant-veille de la guerre dans une formation dont la vocation n'est pas initialement politique, mais qui va fédérer et exprimer le mécontentement croissant de l'opinion serbe : le Club culturel serbe (Srpski kulturni klub, SKK). L'éminent juriste et historien Slobodan Jovanović en est le président.

Mladen J. Žujović s'est exprimé régulièrement dans les colonnes du journal du Club: «Srpski glas» («La Voix serbe»), dont Vasić est le rédacteur en chef, et soustitré : «Jako Srpstvo-Jaka Jugoslavija » (traduisible par « Serbité forte-Yougoslavie forte »). Il tient notamment une chronique consacrée à la situation des fronts guerriers entre 1939 et 1940: Finlande, Norvège, Belgique. Des extraits de son journal de la guerre précédente y sont publiés. Toutefois Žujović s'attache à mettre en relief les caractères nouveaux de la "guerre totale", les armes et tactiques nouvelles, et en particulier l'importance de la propagande. S'il constate que les moyens déployés à Berlin et Rome sont imposants, il n'en soutient pas moins que : «Les décisions sont prises plus vite dans les dictatures mais celles que prennent les gouvernements démocratiques ont davantage d'autorité, elles sont plus fortes. ${ }^{20}$ " Selon lui, les méthodes des régimes autoritaires, de pressions psychologiques, fondées sur l'émotion, appuyées sur un monopole de l'information sont dommageables à terme et vouées à l'échec.

Žujović comprend que le second conflit mondial est engagé autour de "questions idéologiques ", de valeurs et de systèmes politiques antagonistes. En ce qui concerne la Yougoslavie, il considère la redéfinition des intérêts serbes comme l'urgence. Il justifie le besoin d'une formation "transpartisane » nouvelle (le SKK) par la nécessité de faire face aux dérives anti-démocratiques du temps (que les partis seuls ne pourraient enrayer) en même temps que par celle d'un renouveau de cohésion nationale dans ce contexte critique. Il donne comme exemple, dans un article du 11 avril 1940, la détermination de Paul Reynaud et l'idée du nouveau président du Conseil de constituer une union franco-britannique. Démocratie et cadre de l'État-nation lui semblent solidaires, en même temps que de nouvelles modalités de coopération internationale sont requises. L'appartenance de la Yougoslavie au camp des démocraties occidentales ne doit pas faire de doute pour lui.

A Belgrade donc, à la fin de l'été 1941, Žujović s'efforce d'entretenir des liens et d'en tisser de nouveaux. Son jeune frère, Milan, professeur à la Faculté de droit, le seconde dans la capitale occupée. Il rencontre régulièrement Dragoslav Stranjaković et Vojin Andrić du Club culturel serbe, les républicains Vujanac et Prodanović (âgé, le co-fondateur du parti lui dispense des conseils). Plus tard une coopération avec Miodrag Marković du Parti démocrate et Vojislav Grol, le fils de Milan, est initiée, tandis que les contacts avec un représentant du Parti paysan croate de Maček ${ }^{21}$ se poursuivent. Dans un mouvement d'abord d'essence militaire, Žujović défend une marge de manœuvre conséquente pour les civils/politiques. Circulant entre la capitale et une résidence dans la région du Kosmaj (qui ouvre plus au sud sur les collines et reliefs boisés de la Šumadija où les unités de guérilla ont leur cantonnenement), il a aussi en charge les problèmes d'intendance, de ravitaillement et de santé de sa famille. Il est finalement arrêté en mars 1942 par la Gestapo, libéré deux mois plus tard sur intervention d'une de ses relations, faute d'éléments probants à son encontre.

Dans le journal qu'il tient à partir de juin, on observe un suivi régulier de la situation des fronts en Afrique et en Russie principalement. Partant d'une fidélité sans failles à la cause alliée, Žujović fait montre d'une conscience assez nette, dès cette époque, du risque d'un décalage entre la stratégie adoptée par Mihailović (préparation à une 
intervention alliée sans combats prématurés et coûteux pour la population civile) et les intérêts majeurs des "grands alliés". À cet égard, il note que la nomination de Mihailović au grade de général d'armée (en juin) pourrait permettre à Londres d'exiger de lui qu'il passe à l'action quand les Britanniques eux le jugeront nécessaire ${ }^{22}$. locaux sont d'abord méfiants. On craint les menaces sur les biens et la propriété, l'hostilité de principe manifestée « au roi et à la patrie ». Puis, suite aux comportements déplorables de nombre de tchetniks, aux réquisitions croissantes des Allemands que Nedić ne peut tempérer, "les communistes...sans aucun mérite, voient grandir leur réputation et le peuple, de façon assez justifiée, voit en eux les champions de la justice. ${ }^{25}$ » Mais, pour Žujović, un peu plus loin : «En théorie, les communistes ont peut-être raison (par leur activisme) mais la défaite allemande n'aura pas de sens dans un pays désert. ${ }^{26}$ » En effet, le « régime » de représailles des forces d'occupation est très lourd en Serbie : 100 otages serbes tués pour un officier allemand tué, 50 pour un soldat. Cela handicape tout particulièrement une organisation dont quantité de cadres voient leurs responsabilités exposées du fait de la partie publique ou semi-publique du double jeu pratiqué.

Žujović fait état de rumeurs croissantes, dans les villages, sur les passages de locaux et de voisins dans le «camp des partisans ». Il expose, par exemple, la manière avec laquelle un paysan cossu ("najveći gazda ovdašnji»), pensant qu'il est lui-même sympathisant communiste, justifie sa nouvelle adhésion. L'homme lui relate, qu'après 
un séjour en prison à Belgrade, l'égalité éprouvée entre détenus, la solidarité face à l'adversité l'ont entraîné :

« Nous étions deux cents dans la pièce numéro 4 à Banjica ${ }^{27}$, tous ensemble, du Tzigane jusqu'au Roi, médecins, juristes, gens instruits, tous nous appelant camarades et tous respirant comme un seul homme...

Assieds-toi bien confortablement m'sieur Mladen, notre heure arrive. ${ }^{28}$ "

Alors qu'une nouvelle campagne de répression à l'encontre des membres de l'organisation de Mihailović est menée dans la capitale, et que son nom est de nouveau cité dans ce cadre début août 1942, Žujović décide d'interrompre ses allées et venues et de demeurer à la campagne. Il apprend, le 31, que sa maison de famille à Belgrade a été investie par la Gestapo.

Attentif toujours aux informations internationales, il se réjouit des difficultés allemandes à Stalingrad, mais craint que les Allemands soient plus enclins à la répression localement. Ceux-ci semblent, à la mi-octobre, assurés d'avoir démantelé l'organisation à Belgrade. Hébergé chez des amis, de retour dans la capitale, Žujović est décidé à se démettre de ses fonctions, affligé toujours par l'inorganisation et les ambitions personnelles tant dans le Kosmaj qu'à Belgrade. Il envoie des dépêches pour être remplacé, puis fait part de sa démission à Mihailović. Le 19 octobre 1942, il reçoit un courrier qui lui demande de le rejoindre à son quartier général. Il va s'y rendre.

Žujović retrouve le général-ministre ${ }^{29}$ dans le mois qui suit, au nord du Monténégro où il est installé depuis juin. Žujović est nommé en décembre 1942, membre du Comité exécutif du Comité central national (CNK), en compagnie de Dragiša Vasić et Stevan Moljević, créateur avant-guerre d'une section du SKK à Banja Luka en Bosnie occidentale. Au quartier général, il est très proche de Mihailović. Les deux hommes ont des échanges réguliers. Žujović retranscrit notamment les propos acerbes, tenus le 28 février $1943^{30}$, à l'encontre de l'allié britannique, en présence du colonel Bailey et pour lesquels Churchill demandera des explications au gouvernement yougoslave en exil ${ }^{31}$. Début mai 1943, Mihailović le nomme commandant de l' "Armée yougoslave dans la patrie » pour les régions de Bosnie occidentale, de Lika (arrière-pays montagneux de Croatie occidentale où vit une importante population serbe) et de Dalmatie, zone qui se trouve sous domination italienne. Il s'agit de remplacer, à Split, le défunt TrifunovićBirčanin.

Sous pseudonyme, Žujović va œuvrer à élargir l'assise politique du mouvement. Il faut, pour cela, corriger l'image d'une formation exclusivement serbe et menaçant les autres peuples d'une nouvelle hégémonie. Mihailović a lui-même décidé cette mission et choisit un personnage capable de la mener. Jusqu'alors, le gros des Croates favorables à l'union yougoslave (membres du Parti populaire/national yougoslave ou de la société de jeunesse Sokol), relativement bien représentés dans les villes du littoral dalmate, ont affiché leur soutien au leader de l' "Armée yougoslave dans la patrie ». Les écrivains Niko Bartulović et Đura Vilović sont allés à sa rencontre. L'ancien maire de la ville Jakša Račić a rejoint Trifunović-Birčanin en mars 1942. Ils ont formé un "Demokratski blok ».

À Split, Žujović s'évertue aussi à nouer des relations avec les représentants du Parti paysan croate, parti phare dans l'opinion croate et du Parti démocrate indépendant, défenseur traditionnel d'une entente fédérale serbo-croate, influent hors de Serbie. Il prend contact avec des officiers croates à Split et en mobilise une dizaine pour former, dans l'arrière-pays avec une centaine d'étudiants, deux unités croates intégrées à l'armée de Mihailović. La question de l'équipement et de l'armement reste posée avec 
acuité. La dépendance dans laquelle se trouvent les tchetniks locaux vis-à-vis des Italiens (qui ont aidé les Serbes à se défendre contre les Oustachis et les utilisent contre les partisans) et dont Žujović voulait sortir, ne peut être abolie à court terme. Il convient de bénéficier encore d'une aide matérielle tactique, sans compromettre la stratégie. Le nouveau commandant dénonce tout de même ceux qui ont fini par voir dans les occupants «nos "sauveurs ». "

Des difficultés se font jour également avec les communistes qui livrent par voie d'affichage publique les noms des membres de l'organisation de Mihailović. Au sein même de celle-ci, les problèmes sont importants :

"Les vieilles fautes étaient lourdes et toujours mises en évidence contre nous. Ceux qui s'étaient signalés comme "grand-serbes", brutaux, comme adeptes exclusifs de la prétendue "idéologie tchetnik» que Draža (Mihailović) avait condamnée (ce qui ici ne se savait pas), je les ai éliminés. ${ }^{32}$ "

Toutefois, Žujović a le sentiment de progresser. Il fédère et crée un organe civil : le "Comité patriotique ». Mais il est interpellé par les Italiens à la fin du mois de juin 1943, puis est relâché début août. Il poursuit ses entreprises et circule entre Split, l'arrière-pays, Knin et l'Istrie au nord. Sur le mont Dinara, son quartier général est attaqué par les partisans. Des renforts demandés au général-ministre tardent alors à lui parvenir.

41 Le 3 septembre 1943, Mihailović le sollicite pour rencontrer les autorités militaires italiennes en anticipant un désengagement dont les signes avant-coureurs lui sont rapportés. Il s'agit de récupérer armement et troupes éventuelles. Il se rend à Šibenik le lendemain, puis à Zadar, obtient des promesses de ralliements. A l'annonce de la capitulation, le 8 septembre, il proclame la mobilisation générale contre les Allemands en Dalmatie et tente d'éviter l'aggravation du conflit avec les partisans. En définitive, ce sont Allemands et partisans qui profitent le plus du retrait italien. Les uns et les autres progressent en direction de la côte. Sous leurs menaces respectives, Žujović quitte le pays, le 10 à Opatija, en prenant la mer. Il a alors en tête de rejoindre les Britanniques avant d'être réexpédié auprès de son leader. Il est à Brindisi le 16 septembre, puis se rend en Égypte le 3 octobre via Tunis. Au Caire, il entre en relation avec les membres du gouvernement dit « de fonctionnaires» (sans membres des partis) du diplomate Purić, en place depuis le 10 août 1943. Refusant une proposition de l'intégrer, il demeure en contact direct avec Mihailović, qui fait de lui son délégué officiel à l'étranger.

Il faut dire que les travaux des différents cabinets yougoslaves en exil, à Londres d'abord, ont été marqués par l'aggravation des relations serbo-croates, par des différends dans le « camp serbe » entre militaires et civils, fédéralistes et centralistes, " yougoslaves » et " panserbes ", conservateurs et libéraux, sans parler des intrigues de toutes sortes. Le statut de ministre de l'armée de Mihailović a été reconduit, mais la défiance des Britanniques à son égard n'a cessé de croître, tout comme l'agacement à l'égard des exilés. ${ }^{33}$. Le jeune roi Pierre projette de rejoindre Mihailović à son « quartier général de montagne " afin de manifester son attachement et forcer les Britanniques à poursuivre leur soutien. Žujović veut faire de même, mais le général-ministre lui fait savoir dans un message le 26 octobre $1943^{34}$, qu'il préfère un travail d'influence auprès des alliés, là où il se trouve. Le projet du roi échoue. Il subit la pression croissante des autorités britanniques qui veulent remplacer Mihailović. Le désir de mariage du souverain, que la plupart des politiques yougoslaves considèrent comme inopportun, tend également à affaiblir sa position. L'accablement commence à gagner les soutiens 
de Mihailović. Il est qualifié de traître à Moscou devant un congrès panslave à la mioctobre (substitut du Komintern dissous dans l'année) et de "Quisling " par l'agence Reuters début novembre.

À la demande de Ralph Stevenson, ministre britannique auprès de la cour du roi Pierre, Žujović rédige un rapport qui distingue les stratégies politiques de Tito et de Mihailović ${ }^{35}$. Il affirme que le mouvement de celui-ci n'est en rien dominé par une idéologie réactionnaire ou anti-démocratique

«Un des aspects de faiblesse de l'organisation de Mihailović, c'est l'appui sur les unités de l'armée de Nedić. Les tchetniks sont très impopulaires, car ils sont brutaux et parasites, des vauriens. Le peuple ne peut pas leur pardonner la collaboration avec les Allemands. Peutêtre le double jeu serait-il admis si les commandants des unités tchetniks savaient bien se comporter, mais ils sont malhabiles, insuffisamment intelligents. ${ }^{36}$ "

"Tout le monde sait qu'il faudra mener après-guerre de profondes réformes économiques et sociales... En tant que partisan convaincu de la souveraineté électorale populaire dans le sens le plus étendu du terme, et des libertés garanties, Mihailović considère toute organisation politique, économique et sociale comme devant émaner de la volonté générale ${ }^{37}$

ou une volonté hégémonique serbe. Selon lui, après l'annonce des massacres oustachis, n'eut été l'action de Mihailović, tout projet de future Yougoslavie serait devenu inacceptable pour les Serbes. Le général soutient une Yougoslavie fédérale, il a entrepris un ralliement des troupes croates domobranes ${ }^{38}$, annonce des mesures de réparations pour contrecarrer les volontés de vengeance. Il convient ainsi de renforcer son action, notamment en ce qui concerne ses moyens de propagande, pour empêcher des dérives locales " grand-serbes anti-croates ${ }^{39}$. Žujović justifie aussi les politiques de « légalisation » dans l'armée de Nedić et d' « accommodement " avec les Italiens (qu'il critiquait dans son journal auparavant en en pointant les effets pervers). Selon lui, ces politiques se sont imposées dans les régions où pesaient des menaces militaires et " existentielles » très fortes sur la population serbe. L'armée de Nedić serait "prête » désormais à " passer à Mihailović ». Là, où une autonomie militaire et une autosuffisance économique ont été possibles, ces politiques n'ont pas eu de raison d'être, à l'exemple de la Bosnie orientale et du Sandžak. Il reconnaît toutefois que la population a mal admis ce qui est apparu comme un ralliement à la politique de l'occupant. Pour leur part, poursuit-il, les partisans éliminent les élites sociales serbes et ont pour objectif essentiel d'imposer un régime de type bolchévique. Leur action ne peut être qu'antagoniste à celle de Mihailović. Žujović rappelle, qu'en tant que membre du Parti républicain et tout comme les "démocrates de gauche » de la revue " Napred » avantguerre, il a pu éprouver des sympathies pour les communistes (en tant qu'avocat, il les a défendus à plusieurs reprises devant la Cour de sûreté de l'État), mais qu'une rupture "de nature morale» a eu lieu, en septembre 1939, quand leur fidélité à Staline l'a emporté sur tout sentiment de patriotisme ${ }^{40}$.

Selon lui :

"L'esprit international est une chose, l'internationalisme en est une autre... Mihailović considère aussi que la démocratie doit être organisée sur un principe universel et à travers l'étroite coopération de tous les peuples libres. Nos communistes, cependant, ont des objectifs plus modestes, car ils semblent qu'ils se satisfassent à présent d'une bolchevisation des Etats mûrs pour une stricte union avec l'URSS. ${ }^{41}$ "

Le journal tenu par Žujović révèle toutefois de nombreux doutes: sur l'avenir yougoslave, sur la possibilité de contrecarrer la prééminence des intérêts des grandes puissances, sur les innombrables intrigues, sur également, le leadership politique de Mihailović et la communication qu'il adopte à l'égard des Alliés. Il lui semble préférable 
de ne pas cacher la réalité de la situation difficile à Mihailović, comme le font certains responsables. Quand, à la suite de la conférence de Téhéran du 28 octobre au 2 décembre 1943, tout le soutien allié se porte sur Tito, l'accablement de Žujović atteint un paroxysme. Il remet même en question la pertinence du « coup du 27 mars 1941 » et va tenter de trouver appui auprès des agents américains et des forces françaises de la résistance. Le 26 décembre, Žujović propose au roi l'envoi d'une délégation auprès du général De Gaulle à Alger. Purić suggère à Žujović de former lui-même un nouveau gouvernement. Ce dernier refuse. Il n'entend pas non plus partir pour Londres et poursuit son activité en considérant que : «L'essentiel est de tenir. ${ }^{42}$ »

Mihailović réunit un congrès du 25 au 28 janvier 1944, à Ba, près de Ravna Gora. Des représentants de tous les partis serbes, à l'exclusion de l'extrême gauche communiste et de l'extrême droite, s'y associent au " ravnagoristes » hostiles jusque-là aux « vieux partis». Ils constituent ensemble une «Union démocratique populaire/nationale yougoslave». Quelques délégués croates (Vladimir Predavec, fils d'un bras droit de Maček, Bartulović, Vilović et le socialiste Parte), un Slovène et un Musulman y participent. Le Parti paysan croate s'est tenu en réserve. Un projet de fédération de trois entités est défendu. Le général lui-même dénonce les velléités dictatoriales prêtées à Mihailović, ainsi que les vengeances à venir des Serbes ${ }^{43}$. L'union affichée et l'adoption de résolutions démocratiques générales (des litiges et désaccords profonds demeurent entre, notamment, le président socialiste du congrès Živko Topalović et le "ravnagoriste » Stevan Moljević, qui défend prioritairement la constitution d'une entité serbe ethnique étendue) a un double objectif: interne, de rassemblement plus large ; et externe, de reconquête d'un soutien international.

Tenté à nouveau par un retour sur le terrain, Žujović en est dissuadé par le roi. Il se rend ensuite à Gaza, où stationne un contingent de l'armée yougoslave, à Beyrouth où il rencontre un délégué du "Comité français de libération nationale ", des diplomates, militaires et journalistes. La publicité du congrès de Ba est faite dans quelques organes de presse, mais sa tenue ne change pas la donne à l'étranger. De retour au Caire, Žujović a un contact, le 25 février 1944, avec Bailey. Ce dernier, juste rentré de mission, critique "la retenue » de Mihailović. Début mars, Žujović se dit: «Draža doit faire quelque chose activement...les communistes se renforcent de plus en plus » et engage en ce sens le général, critiquant la passivité des troupes près du littoral. Le républicain Jovan Đonović, après des mois de tergiversations, se rend enfin à Alger le 25 mars pour y représenter Mihailović.

Le Roi, finalement marié le 20 mars («contre quelles concessions $?^{44}$ " se demande Žujović), sous le parrainage de son oncle le roi Georges VI, cède aux pressions britanniques. Dans une interview quelques jours plus tard, il semble consentir à reconnaître Tito. Alternent toujours dans l'esprit de Žujović, doutes sur les capacités intrinsèques du mouvement, craintes des progrès de l'Armée rouge, nouveaux espoirs d'un retournement de la politique britannique ou d'une action décisive de Mihailović. Žujović apprend également le décès de son père à Belgrade. Le 1er juin 1944, le roi, démettant Purić de ses fonctions, forme un gouvernement avec le ban/gouverneur de la « Banovine de Croatie » Ivan Šubašić, qu'il charge d'intégrer Tito et son armée. Le 16, Tito entre dans le gouvernement. Le CNK et Mihailović dénoncent cette formation et composent, dans une position désespérée, un cabinet parallèle se prévalant du « roi captif ». 
La position de Žujović, délégué de Mihailović et du CNK, n'est plus tenable au Caire. Il fait établir à Alger une relation radio directe avec Mihailović et, avec l'aide de l'ambassadeur français quitte l'Égypte pour l'Afrique du Nord. Mihailović le charge alors d'organiser des troupes de prisonniers yougoslaves libérés par les Alliés. Il doit s'agir d'en intégrer une partie à la Légion étrangère et de former des unités ("stagiaires " des Français) préparées pour un retour au pays. Les Britanniques s'opposent bien entendu à ce plan. Le 12 septembre 1944, le roi appelle sur RadioLondres tous les combattants à rejoindre l'armée de Tito. Le 22, l'Armée rouge pénètre en Serbie où Mihailović a d'abord tenté de la recevoir en alliée. Le 20 octobre, Soviétiques et partisans de Tito entrent dans Belgrade. Mihailović, à qui le général Wilson a proposé de quitter le sol yougoslave, et qui offre en vain également de se placer sous le commandement des Alliés, passe de Serbie en Bosnie. Il s'évertue à y maintenir des troupes avant de tenter de retourner mener une guérilla en Serbie. Son armée défaite se délite. Il est traqué par la police politique titiste. Tout lien radio avec l'étranger est interrompu le 5 mai 1945. Terré avec quelques hommes, il est capturé en Bosnie orientale, près de Višegrad le 13 mars 1946. Acheminé immédiatement à Belgrade, son procès se déroule à partir du 10 juin. Condamné à mort le 15 juillet, son exécution est annoncée le surlendemain.

Žujović rejoint Paris le 25 novembre 1944, depuis Alger et dans l'avion du général Roger Testard. Engagé dans les activités de l'émigration politique, il échappe en juin 1945 à des agents des services secrets du nouveau pouvoir titiste, chargés de l'enlever, peutêtre de l'éliminer. Accusé lors du même procès que Mihailović, il est condamné à mort par contumace, à la privation de ses droits civils et politiques, à la confiscation de ses biens et à la perte de sa citoyenneté. La pression exercée sur les autorités françaises par Belgrade le contraint à quitter le territoire ${ }^{45}$. Marié à une Française, père depuis peu, il embarque avec les siens et arrive à New York le 16 septembre 1946. De retour à Paris en décembre 1950, il contribue aux travaux d'assistance des réfugiés yougoslaves, œuvre dans les organisations de l'émigration et intègre $\mathrm{l}^{\prime}$ " Institut de science économique appliquée » en tant que bibliothécaire. Plus tard il est nommé chargé de recherches. Apatride encore, il meurt à Paris, le 15 novembre 1969.

\section{NOTES}

1. Petranović, Branko, Srbija u drugom svetskom ratu 1939-1945, Beograd, 1992, p. 76.

2. Ibid., page 108 .

3. Terzić, Velimir, Jugoslavija u Aprilskom ratu 1941, Titograd, 1963, pp. 575-576. Outre une nette supériorité technique, notamment pour l'aviation, l'artillerie lourde et les blindés, la Wehrmacht compte 870000 hommes pour environ 600000 Yougoslaves.

4. En serbe, «prendre le maquis » se dit : « partir dans la forêt » (« u šumu »).

5. Chef de guerre ; titre honorifique traditionnel.

6. Živanović Sergije M., Treći srpski ustanak 1941, knj.1, Čikago, 1962, p. 66. Mihailović a participé à cette importante victoire serbe en 1914. 
7. Karchmar Lucien, Draža Mihailović and the rise of the četnik movement 1941-1942, New YorkLondres, 1987, p. 82.

8. Archives de Yougoslavie : АРХИВ ЈУГОСЛАВИЈЕ, ЕМИГРАНТСКА ВЛАДА, 103-5-51 in НИКОЛИТ КОСТА, ИСТОРИЈА РАВНОГОРСКОГ ПОКРЕТА, vol. 2, БЕОГРАД, 1999, p. 353.

9. Žujović Mladen J., Ratni dnevnik 2, Jugoslavija u II svetskom ratu, Vrnjačka Banja, 2004, p. 9.

10. Žujović Mladen J., Eseji o ljudima i događajima, Vrnjačka Banja, 2004, p. 79. Žujović relate l'accueil, le 19, chez Boško Novaković, « un Tchetnik de la dernière guerre » qui a appris la mort de son fils au front à skadar, il y a quelques jours, et refuse d'envisager que la guerre soit terminée. Il provoque, chez Vasić et Žujović, un sentiment de révolte contre la résignation, et de devoir d'encadrer une lutte de libération à venir.

11. Mihailović se souvient lors de l'interrogatoire avant son procès, qu'il a lui-même engagé la formation de l'instance politique. Сf. РАТ И мИР ЂЕНЕРАЛА II, "Choix de textes de guerre ", Beograd, 1998, p. 499.

12. Žujović, « Ratni dnevnik 2 », op. cit., p. 9.

13. Žujović, Eseji... , op. cit., p. 87.

14. Petranović, op. cit., p. 163.

15. Ibid., p. 146.

16. Žujović, « Ratni dnevnik 1 », op. cit., p. 6.

17. Žutić Nikola, Liberalizam i Srbi u prvoj polovini XX vijeka, Beograd, 2007, p. 59.

18. Domaine attribué à un cavalier ottoman. Une des formes de la propriété dans l'ex-empire.

19. Žutić, op. cit., p. 59.

20. «Srpski glas », publié par Stijović Milun, Novi Sad, $2004 ; n^{\circ}$ 22, 11 avril 1940.

21. Žujović, « Ratni dnevnik 2 », op. cit., p. 60.

22. Ibid., p. 14.

23. Ibid., p. 21.

24. Ibid., p. 24, c'est-à-dire personnages enclins à se soumettre et répondre « amen ».

25. Ibid., p. 25.

26. Ibid., p. 32.

27. Quartier en périphérie de Belgrade où est installé un des principaux camps d'internement.

28. Ibid., p. 35.

29. Mihailović nommé ministre de l'Armée du gouvernement yougoslave en exil, en janvier 1942. 30. Fâché contre les demandes d'action des Britanniques, contraires à sa stratégie de préparation, et le peu d'armes qu'ils lui font parvenir, Mihailović tient, à l'occasion d'une fête locale au Monténégro, le 28 février 1943, en présence du colonel Bailey, un discours où il dit : « Depuis que la France n'est plus là c'est comme si nous n'avions plus personne au monde... Oh, nous sommes seuls, chers amis, nos alliés ressemblent à des marchands de viande humaine... Ils considèrent que nous n'avons pas assez perdu de sang et qu'il faut que nous nous épuisions dans des actions sans aucun intérêt militaire qui vont causer pourtant la mort de centaines et de milliers de Serbes. » Cf. «Ratni dnevnik 3 », pp. 357-358. Bailey répond à Mihailović et rend compte ensuite à Churchill.

31. Le journal tenu par Žujović, de novembre 1942 à août 1943, a été pris par les partisans à l'issue d'un affrontement au nord-est de Split en août 1943. Des informations sur la période et notamment ce texte du 28 février figurent dans un «Zbornik dokumenata " (Recueil de documents ») qui, avec les «Journaux de guerre » (Première et Seconde Guerre mondiale), le volume déjà mentionné : « Esej o ljudima i događajima », composent la somme publiée en 2004, à Vrnjačka Banja, en Serbie, à partir d'archives familiales et de celles du « Hoover Institution Archives » de Stanford en Californie.

32. Žujović, « Ratni dnevnik 2 », op. cit., p. 94. 
33. D'autre part, les victoires de la Neretva et de la Sutjeska (mars et avril 1943), le recrutement important suite à la capitulation italienne en septembre, font des partisans de Tito, l'acteur majeur sur qui comptent le plus les Britanniques pour affaiblir les Allemands dans la région.

34. Žujović, « Ratni dnevnik 2 », op. cit., p. 145.

35. Žujović, Zbornik dokumenata, op cit., pp. 251-316.

36. Ibid., p. 21.

37. Ibid., p. 311.

38. "défenseurs de la patrie»; force territoriale composée d'enrôlés par conscription obligatoire.

39. Ibid., p. 266.

40. Ibid., p. 311.

41. Ibid., p. 315.

42. Žujović, « Ratni dnevnik 2 », op. cit., p. 215.

43. Des cycles de vengeances, des massacres ont déjà atteint les milices et la population musulmanes de l'est de la Bosnie principalement, en réaction notamment aux exactions des Oustachis auxquels ont pris part certaines de ces milices.

44. Žujović, Ratni dnevnik 2, op. cit., p. 325.

45. Un article de l'Humanité, le 30 mars 1946, est titré : « Mihailović est en prison, ses agents sont à Paris? » Žujović, « Ratni dnevnik 3 », op. cit., p. 318.

\section{RÉSUMÉS}

Mladen J. Žujović, un cas à part: un réformateur qui rejoint le mouvement de résistance de Mihailović, tout en critiquant les exactions des Tchekniks

Cette communication traite des différents aspects et moments de l'action politique de Mladen J. Žujović, en Yougoslavie puis auprès des Alliés au Moyen-Orient, entre 1941 et la fin de 1945. Militant avant la guerre au Parti Républicain yougoslave qui se voulait à la fois libéral, pacifiste, partisan d'une réforme agraire et de la protection des travailleurs, décidé à ne pas accepter la défaite et à continuer la lutte contre l'occupant, il rejoint Mihailović dès l'été 1941 et, contraint de quitter son pays à la fin de 1943, le représente auprès des Britanniques. Il n'hésite pas à critiquer les exactions des Tchekniks, les divisions et la confusion du camp de Mihailović, il tente, à Split, de gommer les aspects trop serbes du mouvement, pour obtenir la participation de Croates et de musulmans, mais il reste opposé au communisme des partisans. Les Alliés ayant choisi de soutenir Tito, il se retrouve dans le camp des vaincus ; rejoignant la France, il condamné à mort par contumace en juillet 1945.

This communication deals with different aspects and stages of the political activities of Mladen J. Žujović, in Yugoslavia and then on the part of the allies in the Middle East, between 1941 and the end of 1945. Member before the war of the Yugoslavian Republic Party, which considered itself liberal and pacifist and which favored agrarian reform and the protection of workers, he resisted defeat and continued the struggle against the occupation.To that end he rejoined Mihailović in summer 1941 and, obliged to flee his country at the end of 1943, he represented it in England. He did not hesitate to criticise the demands of the Tchetniks, the divisions and the confusion of the Mihailović camp. In Split, he tried to erase the overly Serbian aspects of the movement, in order to secure the participation of the Croats and muslims, but he remained opposed to the 
communism of the partisans. When the allies threw their weight behind Tito, he found himself on the losing side. Upon his return to France, he was sentenced to death by default in July 1945.

INDEX

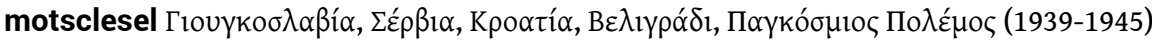

Index chronologique : guerre mondiale (1939-1945)

Thèmes : Histoire

motsclesmk ЈУГОСЛАВИЈА, БЕЛГРАД, СРБИЈА, ХРВАТСКА

Keywords : Mihailović Dragoljub (1893-1946), serbian nationalism, Tchetniks, Žujović Mladen J. (1895-1969), Serbia, Croatia, Belgrade, History

Mots-clés : Mihailović Dragoljub (1893-1946), Banovine de Croatie, CNK, nationalisme serbe, Cvetković Dragisa (1893-1969), Domobranes, Tchetnik, résistance yougoslave, Kalafatović Danilo (1875-1946), Kosmaj, Mihailović Dragoljub (1893-1946), Mirković Borivoj (1884-1969), Oustachi, Šumadija, Tcheknik, Žujović Mladen J. (1895-1969)

motsclestr Yugoslavya, Sırbistan, Hırvatistan, Belgrat, Dünya savaşı (1939-1945)

Index géographique : Serbie, Yougoslavie, Croatie, Belgrade

\section{AUTEUR}

ROLAND VASIC

INALCO CREE EA 4513 